\title{
Cancer as a Continuum: a Literature Review and a Biological Interpretation
}

\author{
Oddone $\mathbf{E}^{1}$, Crosignani $\mathbf{P}^{1}$, Modonesi $\mathbf{C}^{2, *}$ \\ ${ }^{1}$ Department of Public Health, Experimental and Forensic Medicine, University of Pavia, 27100 Pavia, Italy \\ ${ }^{2}$ Natural History Museum, Department of Biosciences, University of Parma, 43100 Parma, Italy \\ *Corresponding Author: modonesi@unipr.it
}

Copyright @ 2013 Horizon Research Publishing All rights reserved.

\begin{abstract}
Basic research shows that the genetic control of development cannot fully explain the phenotypic plasticity of humans and other metazoans. This challenges some tenets of the conventional paradigm of life sciences based on DNA, restoring a role of the environment in biological processes like the regulation of development, cell differentiation and disease. The environment - in a broad sense - affects biological phenotypes throughout the entire lifespan and can induce cancer, its progression and recurrence as well as its reversal. This is very important in cancer epidemiology as it implies that environmental exposures can be considered both as risk (or protective) and prognostic factors. This review integrates information from epidemiologic and biological research. We studied the impact of hazardous and protective exposures, lifestyles, and diet on the survival of patients who had been diagnosed with cancer.We selected studies from two digital databases, using a few combinations of key terms, namely overall survival, cancer-specific survival, recurrence and quality of life (QoL). Survival and/or recurrence were expressed as hazard or risk ratios, as second cancer diagnosis and as indicators of QoL such as performance status. We found 53 articles indicating that risk and protective factors can also influence prognosis after cancer diagnosis. Cigarette smoking and, among protective factors, diet and physical activity are the exposures most frequently investigated after a diagnosis of cancer.
\end{abstract}

Keywords Risk Factors, Exposure Cessation, Prognosis, Post-Diagnosis, Prevention, Cancer Models, TOFT, Theoretical Biology

\section{Introduction}

Genetic, epigenetic and organism research has illustrated the limits of explaining cancer initiation and progression as due to a microscopic "cause" in the genetic make-up of a cell. The assumption that the earliest steps of cancer development occur at the gene and cell levels is no longer taken for granted. This attitude about the theoretical foundation of cancer biology is spreading so widely in the biomedical community that some authors have suggested that cancer research is approaching the so-called "paradigm instability" [1]. In other words, the traditional methods of studying and explaining the biology of the disease may have to be radically reformed or replaced.

Summarizing the state of the art in general terms, two main approaches compete to provide a scientific reconstruction of carcinogenesis (Figure 1). The first reflects the traditional paradigm of molecular biology by stating that cancer is a genetic disease [2]. The so-called Somatic Mutation Theory (SMT) epitomizes this approach, though some variants have been proposed to extend the possible causes of malignant transformation of the cell to non-genetic factors [1]. Rather than focusing on structural alteration of DNA, these variants focus on functional differences in gene expression, especially due to epigenetic alterations, such as DNA methylation, chromatin remodeling, and histone acetylation. By adopting this alternative approach, the role of the extracellular, micro-environmental changes is re-directed toward molecular events taking place mostly within the cell. In this way, cancer-environment links become focused on the effects of carcinogens on genes - even when many carcinogens are not genotoxic [3] — or other cellular structures.

\begin{tabular}{|c|c|c|}
\hline Essentials of each approach & SMT & TOFT \\
\hline Assumptions & $\begin{array}{l}\text { Quiescence is the default state of metazoan epithelial } \\
\text { cells } \\
\text { The single cell is the target of carcinogens }\end{array}$ & $\begin{array}{l}\text { Proliferation is the default state of metazoan epithelial } \\
\text { cells } \\
\text { Tissue is the target of carcinogens }\end{array}$ \\
\hline Cancer causation & Bottom-up causation (linear causation) & $\begin{array}{l}\text { Bottom-up and top-down causation (circular } \\
\text { causation) }\end{array}$ \\
\hline Cancer disease & Due to the loss of control of cell proliferation & Due to tissue disruption \\
\hline Implications & $\begin{array}{l}\text { Cell growth is out of the organism's control } \\
\text { Cancer is a linear, irreversible process }\end{array}$ & $\begin{array}{l}\text { Cancer is not independent from organism's control } \\
\text { Cancer is a reversible process }\end{array}$ \\
\hline
\end{tabular}

Figure 1. Features of the Somatic Mutation Theory (SMT) and Tissue Organization Theory (TOFT). Modified from Soto and Sonnenschein [78] 
The second approach, largely developed within a systems biology perspective, considers carcinogenesis as a multicellular process, formalized by the Tissue Organization Field Theory (TOFT). According to this theory, the biological development of multicellular systems involves an interplay between inside and outside cellular environments where a set of multifactorial dynamics and processes entail some risks, such as cancer and other developmental pathologies $[4,5]$. From a TOFT perspective, carcinogenesis is promoted by means of external influences on the stromal micro-environment [6].

Experimental studies have shown that the initial steps of cancer development depend on changes in the way cells interact with each other and their surroundings. They also indicate that cancer cells can reverse their malignant phenotype when the tissue micro-environment is altered [7-13]. Thus, cancer progression is not irreversible and can be modified by restoring proper signals in the cross-talk between the micro-environment and the cancer cells; that is to say, by restoring the "normal" morphogenetic field of a tissue.

Herein, we evaluate data from 53 epidemiologic studies on cancer patients with the purpose of translating evidence on cancer risk into prognostic information while applying a biological, non-deterministic perspective to the interpretation of epidemiologic data. We call this approach "cancer as a continuum", as the same factors act both as risk factors and determinants of prognosis.

We reviewed studies that investigated the impact of risk and protective factors on the survival of cancer patients. We have used the terms 'risk factors' and 'protective factors' to mean factors related to environmental exposures, lifestyles and habits that influence human cancer risk.

\section{Materials and Methods}

We made a qualitative review of the literature. The basic requirement for selecting articles was that they focused on comparisons of patients who continued and patients who stopped being exposed to risk factors after a cancer diagnosis. We also included articles that compared patients who were exposed and those who were not exposed to protective factors after a cancer diagnosis.

The papers were selected using two digital databases PubMed (U.S. National Library of Medicine, National Institutes of Health) and Embase - according to a strategy designed to identify relevant research articles and reviews. We set no time limits that would reduce the range of our search or collect only recent articles.

We selected articles using a small number of key terms, in combinations; examples are 'cancer determinants', 'exposure cessation', 'diet', 'risk factors', 'prognosis', 'post-diagnosis', and 'survival'. We also checked the literature cited and listed in the selected studies' references and included any that met our criteria in the analyses.

Among the different end-points may be used to assess the prognosis of cancer patients and to make inferences on their survival, we selected overall survival, cancer-specific survival, recurrence and QoL as end-points. The measures of survival and/or recurrence were expressed as hazard ratio or risk ratio, and as second cancer diagnosis.

Table 1. General overview of studies included in the review

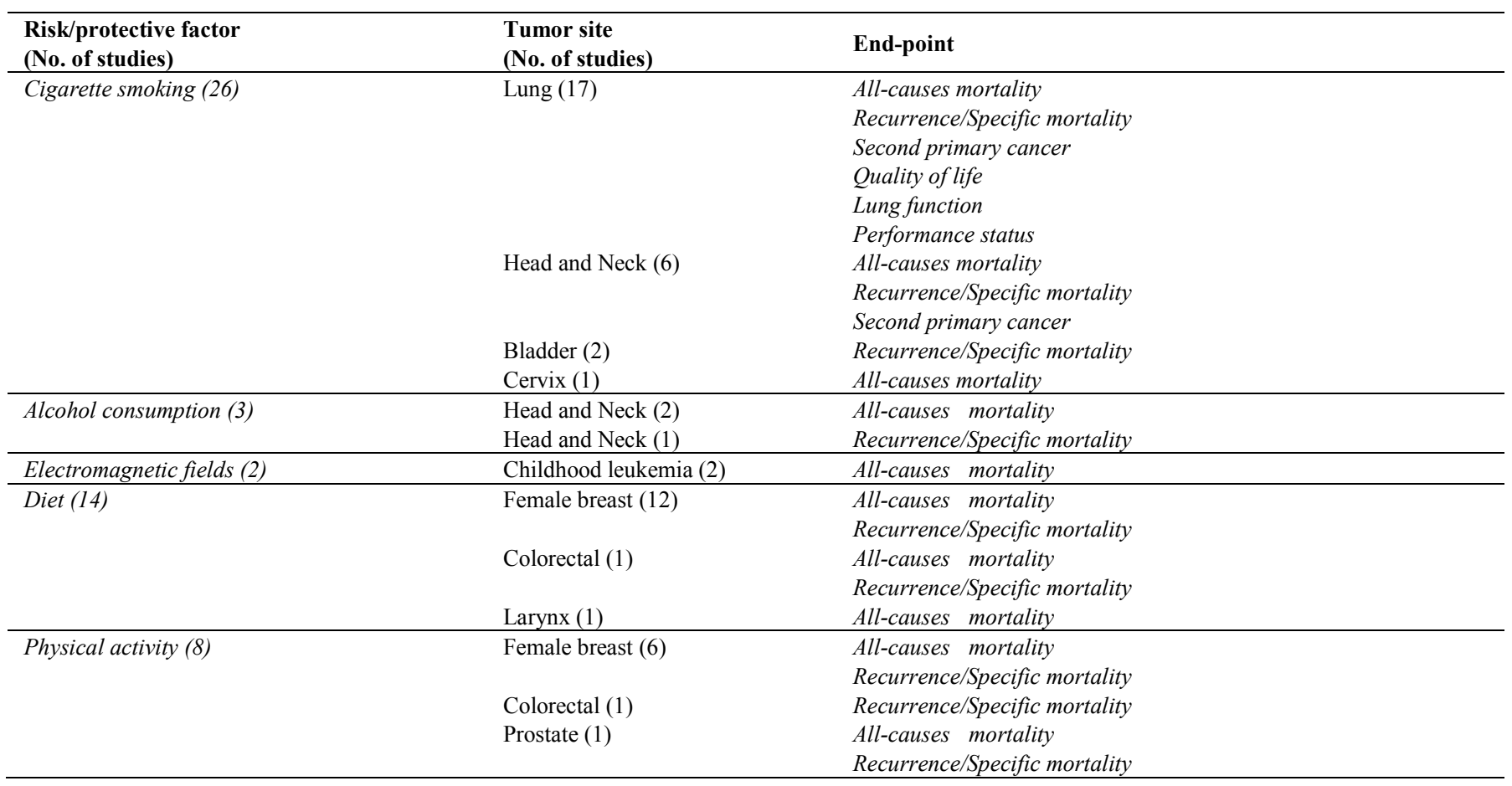




\section{Results}

Fifty three articles met our search criteria (Table 1). Cigarette smoking was the exposure most frequently investigated as risk factor after a diagnosis of cancer. Seventeen scientific articles examined a possible role of post-diagnosis smoking habits on the prognosis of lung cancer, QoL and pulmonary function; six focused on the relationship between post-diagnosis smoking and the prognosis of head and neck cancers; two analyzed the same relationship for bladder cancer and one studied the influence of nicotine abuse on the therapy of cervix carcinoma. Two articles investigated the relation between all-causes mortality and second cancer with alcohol consumption in head and neck cancer patients and one looked at the relationship between post-diagnosis alcohol consumption and specific mortality from breast cancer; two analyzed general mortality in children with leukemia continuing to be exposed to electromagnetic fields (EMF). Finally, a number of studies dealt with the possible positive roles of diet and physical activity on cancer prognosis: 12 studied the effects of diet on patients with breast cancer; one the effects of diet on patients with colorectal cancer; one the effects of diet on patients with larynx cancer; six looked at the effects of physical activity on patients with breast cancer; one the effects of physical activity on patients with colorectal cancer; one the effects of physical activity on patients with prostate cancer.

Regarding lung cancer, several articles analyzed the possible risk of continuing smoking on different outcomes, such as all-causes mortality, recurrence, second primary cancer, QoL and pulmonary function (Table 2). The overall survival of lung cancer is generally shorter for patients who continue to smoke after the diagnosis [14-16] though one study did not describe any increase in risk [17]. Carrying on smoking during chemo-radiotherapy or before surgery is associated with a poorer prognosis and increased mortality $[18,19]$.

Male sex also seems to be related to higher mortality from lung cancer, in patients who continue to smoke and those who stop at diagnosis [20]. The adverse effect on survival is observed equally for small-cell and non-small cell lung cancer, with a stronger correlation with the latter [21].

Despite the lack of statistical significance in some results $[18,19]$, lung cancer recurrence too seems to be related to not stopping smoking after diagnosis [16], and the increase in risk is consistent in small-cell and non-small cell lung cancer [21].

Several studies considered second primary cancers in lung cancer patients as a further outcome. Strong increases in risk are reported (in patients who do not quit smoking after diagnosis of the first lung cancer) for all malignant cancers [22-25] but also specifically smoking-related cancers [24] or a second lung cancer $[23,26]$. This effect seems to be present both for small-cell and non-small cell lung cancers [21].

In addition to these considerations, continued smoking leads to a worse QoL for lung cancer patients [27,28] and to loss of lung function [29].

Cardio-respiratory deaths alone did not account for the poor survival of patients who continued to smoke. But poor survival could be referred to an increased likelihood of cancer progression [21]. Moreover, continuing smokers did not have a higher incidence of toxicity related to treatment breaks, but those who required an interruption in treatment had poorer overall survival [18].

Cigarette smoking is a well-known risk factor not only for lung cancer: head and neck cancers, bladder and cervix cancer recognize smoking as a contributory cause. Some studies highlighted a role of smoking habits after diagnosis of head and neck cancer in terms of shorter survival [30,31], recurrence [31,32], poorer response to radiation therapy [30], and second primary cancer [33], although some estimates did not reach the conventional level of statistical significance $[34,35]$.

Similarly, two studies analyzed the role of continuing smoking after diagnosis of bladder cancer and one study centered on the same relationship for cervix carcinoma. Bladder cancer seems to recur more frequently in patients who continue smoking [36,37] and cervix carcinoma patients who smoke during radiation treatment have shorter survival, especially those in the early stages [38].

Besides cigarette smoking, alcohol consumption is also considered a risk factor. This habit can lead to a worse prognosis if continued after diagnosis. We found three articles that investigated this subject (Table 3).

Postmenopausal women who do not stop smoking seem to have a higher risk of recurrence of breast cancer [39] and cancer patients more frequently had a second primary cancer [33] and shorter survival [35], though not all studies are conclusive about the latter [32].

Recent years have seen increasing evidence of a causal role of magnetic field exposure in childhood leukemia [40]. Two studies (Table 3) describe the effect of continuing exposure to residential magnetic fields in the disease progression, finding a substantial clear effect on overall survival, although the influence on recurrence is of borderline significance $[41,42]$.

Finally, if some risk factors can also act as worsening prognostic factors, it is reasonable to assume that protective factors could improve prognosis: this is the case of diet and physical activity. Several studies have suggested that food habits can play an important role in increasing cancer risk [43]. It has also become increasingly clear that certain foods, such as legumes and other vegetables, can be important in reducing the risk [44]. Today diet is also considered a determinant of survival in cancer patients. It is fast becoming apparent that prudent dietary habits can help improve overall and specific survival in breast cancer patients [45-47]. Meyerhardt and colleagues [48] reported similar results on overall and colorectal survival. 
Table 2. Studies dealing with cigarette smoking by end-point and cancer site

\begin{tabular}{|c|c|c|c|c|c|}
\hline $\begin{array}{l}\text { End Point / } \\
\text { Cancer Site }\end{array}$ & Author (year) & Study design & Estimate $(95 \% \mathrm{CI})$ & $p$-value & Note \\
\hline \multicolumn{6}{|c|}{ Cigarette smoking } \\
\hline \multicolumn{6}{|c|}{ All-causes mortality } \\
\hline \multirow[t]{14}{*}{ Lung } & $\begin{array}{l}\text { Johnston-Early A et al. (1980) } \\
14\end{array}$ & Cohort & Survival difference & 0.035 & \\
\hline & Videtic G et al. (2003) ${ }^{18}$ & Cohort & Log-rank & 0.0017 & \\
\hline & Sardari Nia P et al. (2005) ${ }^{19}$ & Cohort & RR $0.45(0.21-0.97)$ & 0.042 & Non-smokers before surgery \\
\hline & & & RR $0.54(0.35-0.83)$ & 0.006 & $\begin{array}{l}\text { Former smokers before } \\
\text { surgery }\end{array}$ \\
\hline & & & RR 0.34 (0.16-0.70) & 0.004 & Recent quitters before surgery \\
\hline & & & RR 1 (referent) & . & Current smokers \\
\hline & Videtic G et al. $(2005)^{20}$ & Cohort & Log-rank & 0.0046 & Sex and smoking status \\
\hline & Baser S et al. (2006) ${ }^{17}$ & Cohort & Log-rank & 0.64 & \\
\hline & Saito-Nakaya K et al. (2006) ${ }^{15}$ & Cohort & HR $6.4(1.7-23.9)$ & 0.005 & \\
\hline & Chen J et al. (2010) ${ }^{16}$ & Cohort & HR 1 (referent) & $<0.01$ & Never quitters \\
\hline & & & HR $0.73(0.53-1.01)$ & . & $\begin{array}{l}\text { Quitters } \geq 1 \text { year or never } \\
\text { smokers }\end{array}$ \\
\hline & & & HR $0.54(0.37-0.77)$ & . & Quitters at or after diagnosis \\
\hline & Parsons A et al. $(2010)^{21}$ & Meta-analysis & HR $2.94(1.15-7.54)$ & . & Non-small cell lung cancer \\
\hline & & & HR $1.86(1.33-2.59)$ & . & Small-cell lung cancer \\
\hline \multicolumn{6}{|c|}{ Head and Neck } \\
\hline & Browman GP et al. $(1993)^{30}$ & Cohort & RR $2.3(1.2-4.2)$ & 0.008 & $\begin{array}{l}\text { Smoking during radiation } \\
\text { therapy }\end{array}$ \\
\hline & Browman GP et al. $(2002)^{34}$ & Cohort & HR $1.17(-)$ & 0.07 & $\begin{array}{l}\text { Smoking during radiation } \\
\text { therapy }\end{array}$ \\
\hline & Chen AM et al. $(2011)^{31}$ & Case-Control & Log-rank & $<0.001$ & $\begin{array}{l}\text { Smoking during radiation } \\
\text { therapy }\end{array}$ \\
\hline & Mayne ST et al. (2009) ${ }^{35}$ & Cohort & RR 1.83 (0.85-3.94) & . & \\
\hline Cervix & Kucera H et al. $(1987)^{38}$ & Cohort & Survival difference & $<0.01$ & Smoking at diagnosis \\
\hline
\end{tabular}

Table 2- Continued

\begin{tabular}{|c|c|c|c|c|c|}
\hline $\begin{array}{l}\text { End Point / } \\
\text { Cancer Site }\end{array}$ & Author (year) & Study design & Estimate $(95 \% \mathrm{CI})$ & $p$-value & Note \\
\hline \multicolumn{6}{|l|}{$\begin{array}{l}\text { Recurrence Specific } \\
\text { mortality }\end{array}$} \\
\hline \multirow[t]{4}{*}{ Lung } & Videtic G et al. (2003) ${ }^{18}$ & Cohort & HR $1.26(1.06-1.50)$ & . & Recurrence \\
\hline & Sardari Nia P et al. (2005) ${ }^{19}$ & Cohort & HR $1.86(1.01-3.41)$ & . & Recurrence \\
\hline & Chen J et al. (2010) ${ }^{16}$ & Cohort & HR 1 (referent) & . & Never quitters, recurrence \\
\hline & & & HR $0.59(0.34-0.98)$ & . & $\begin{array}{l}\text { Quitters at or after diagnosis, } \\
\text { recurrence }\end{array}$ \\
\hline \multirow[t]{2}{*}{ Head and Neck } & Stevens MH et al. $(1983)^{32}$ & Cohort & RR $4.0(-)$ & . & Recurrence \\
\hline & Browman GP et al. (2002) ${ }^{34}$ & Cohort & HR $1.23(-)$ & 0.06 & $\begin{array}{l}\text { Smoking at baseline, } \\
\text { cancer-related deaths }\end{array}$ \\
\hline
\end{tabular}




\begin{tabular}{|c|c|c|c|c|c|}
\hline & Browman GP et al. (2002) ${ }^{34}$ & Cohort & OR $1.08(-)$ & 0.65 & $\begin{array}{l}\text { Smoking during therapy, local } \\
\text { recurrence }\end{array}$ \\
\hline & Chen AM et al. (2009) ${ }^{31}$ & Case-control & Log-rank & 0.01 & \\
\hline \multirow[t]{4}{*}{ Bladder } & Fleshner N et al. (1999) ${ }^{36}$ & Cohort & HR 1 (referent) & . & Ex smokers, recurrence \\
\hline & & & HR $0.99(0.77-1.25)$ & 0.89 & Quitters, recurrence \\
\hline & & & HR 1.40 (1.03-1.91) & 0.03 & Continued smokers, recurrence \\
\hline & Chen CH et al. (2007) ${ }^{37}$ & Cohort & HR $2.2(1.2-4.0)$ & 0.01 & Recurrence \\
\hline \multicolumn{6}{|l|}{ Second primary } \\
\hline \multirow[t]{6}{*}{ Lung } & Richardson GE et al. (1993) ${ }^{26}$ & Cohort & RR 32 (12-69) & . & $\begin{array}{l}\text { After non-small cell lung } \\
\text { cancer }\end{array}$ \\
\hline & Yoshida T et al. $(1996)^{22}$ & Cohort & RR $3.2(1.2-6.9)$ & . & After small-cell lung cancer \\
\hline & Tucker MA et al. (1997) ${ }^{23}$ & Cohort & SIR $17(11-26)$ & . & $\begin{array}{l}\text { Lung cancer after small-cell } \\
\text { lung cancer }\end{array}$ \\
\hline & Kawahara M et al. $(1998)^{24}$ & Cohort & RR $4.3(1.1-15.9)$ & 0.03 & After small-cell lung cancer \\
\hline & Kawaguchi T et al. (2006) ${ }^{25}$ & Cohort & RR $5.2(1.6-11.7)$ & . & $\begin{array}{l}\text { After non-small cell lung } \\
\text { cancer }\end{array}$ \\
\hline & Parsons A et al. $(2010)^{21}$ & Meta-analysis & HR $1.86(0.96-3.60)$ & 0.775 & After small-cell lung cancer \\
\hline Head and Neck & Do KA et al.(2004) ${ }^{33}$ & Cohort & RR 2.75 (1.49-5.07) & . & \\
\hline \multicolumn{6}{|l|}{ Quality of life } \\
\hline \multirow[t]{2}{*}{ Lung } & Garces YI et al. (2003) ${ }^{27}$ & Cohort & LCSS score & $<0.0001$ & \\
\hline & Chen J et al. $(2012)^{28}$ & Cohort & $\begin{array}{l}\text { Analysis of variance } \\
\text { of } 5 \text { groups }\end{array}$ & 0.0382 & \\
\hline
\end{tabular}

Lung function

Lung

Nieder $\mathrm{C}$ and Bremnes $\mathrm{RM}$ $(2008)^{29}$

Review

Performance status

Lung

Baser S et al. (2006) ${ }^{17}$

Cohort

OR $7.09(1.99-25.3) \quad 0.002$

OR $6.99(1.76-27.7) \quad 0.006$

Worst PS six months from diagnosis

Worst PS six months from diagnosis

Table 3. Studies dealing with alcohol consumption and low-frequency magnetic fields by endpoint and cancer site

\begin{tabular}{|c|c|c|c|c|c|c|}
\hline $\begin{array}{l}\text { End Point / } \\
\text { Cancer Site }\end{array}$ & Author (year) & Study design & Estimate $(95 \% \mathrm{CI})$ & $\begin{array}{l}p \text {-valu } \\
\text { e }\end{array}$ & Note & \\
\hline \multicolumn{7}{|c|}{ Alchol Consumption } \\
\hline \multicolumn{7}{|c|}{ All causes mortality } \\
\hline Head and Neck & Mayne ST et al. (2009) ${ }^{35}$ & Cohort & RR $2.72(1.20-6.14)$ & . & High alcohol intake & \\
\hline \multicolumn{7}{|c|}{$\begin{array}{l}\text { Recurrence/Specific } \\
\text { mortality }\end{array}$} \\
\hline Female Breast & Kwan ML et al. (2009) ${ }^{39}$ & Cohort & HR 1.19 (1.01-1.40) & . & $\begin{array}{l}\text { Alcohol intake } \\
\text { postmenopausal women }\end{array}$ & \\
\hline \multicolumn{7}{|l|}{ Second Primary } \\
\hline Head and Neck & Do KA et al. $(2003)^{33}$ & Cohort & $\operatorname{RR} 1 \cdot 3(1 \cdot 0-1 \cdot 7)$ & . & High alcohol intake & \\
\hline
\end{tabular}




\begin{tabular}{|c|c|c|c|c|c|}
\hline $\begin{array}{l}\text { Low Frequency } \\
\text { Magnetic Fields }\end{array}$ & & & & & \\
\hline \multicolumn{6}{|l|}{ All causes mortality } \\
\hline \multirow[t]{2}{*}{ Childhood leukemia } & Foliart DE et al. $(2006)^{41}$ & Cohort & \multicolumn{2}{|c|}{ HR $4.53(1.49-13.76)$} & \\
\hline & Svendsen AL et al. (2007) ${ }^{42}$ & Cohort & \multicolumn{2}{|c|}{ HR $3.0(0.9-9.8)$} & \\
\hline $\begin{array}{l}\text { End Point/ } \\
\text { Cancer Site }\end{array}$ & Author (year) & $\begin{array}{l}\text { Study } \\
\text { design }\end{array}$ & Estimate $(95 \% \mathrm{CI})$ & $p$-value & Note \\
\hline \multicolumn{6}{|l|}{ Diet } \\
\hline \multicolumn{6}{|l|}{ All causes mortality } \\
\hline \multirow[t]{12}{*}{ Female breast } & Shu XO et al. (2009) ${ }^{54}$ & Cohort & HR $0.71(0.54-0.92)$ & . & Soy food intake \\
\hline & Ingram D (1994) ${ }^{49}$ & Cohort & Log-rank & 0.0206 & High fruit consumption \\
\hline & McEligot AJ et al. (2006) ${ }^{50}$ & Cohort & HR 3.12 (1.79-5.44) & . & Total fat \\
\hline & & & HR $0.48(0.27-0.86)$ & & Fiber intake \\
\hline & & & HR 0.57 (0.35-0.94) & & Vegetable intake \\
\hline & & & HR $0.63(0.38-1.05)$ & & Fruit intake \\
\hline & McCann SE et al. $(2003)^{86}$ & Cohort & HR $0.49(0.26-0.91)$ & trend 0.02 & Soy food intake \\
\hline & Beasley JM et al. (2011) ${ }^{87}$ & Cohort & HR 1.41 (1.06-1.87) & trend 0.03 & High saturated fat intake \\
\hline & & & HR 1.78 (1.35-2.32) & trend 0.01 & High trans fat intake \\
\hline & Kwan ML et al. (2009) ${ }^{39}$ & Cohort & HR 0.57 (0.36-0.90) & trend 0.02 & Prudent dietary pattern \\
\hline & Holmes MD et al. (1999) ${ }^{51}$ & Cohort & RR $0.65(0.47-0.88)$ & . & High protein intake \\
\hline & Kang X et al. $(2010)^{55}$ & Cohort & RR 0.88 (0.56-1.24) & trend 0.76 & $\begin{array}{l}\text { Soy food intake, postmenopausal } \\
\text { women }\end{array}$ \\
\hline Colorectal & ${ }_{48}^{\text {Meyerhardt JA et al. (2007) }}$ & Cohort & HR 2.32 (1.36-3.96) & $\begin{array}{l}\text { trend } \\
<0.001\end{array}$ & Risk for western dietary pattern \\
\hline \multirow[t]{8}{*}{ Larynx } & Crosignani $\mathrm{P}$ et al. (1996) ${ }^{44}$ & Cohort & HR $0.50(0.30-0.83)$ & . & High meat (beef, veal) intake \\
\hline & & & HR $0.54(0.32-0.90)$ & . & High bread intake \\
\hline & & & HR $0.59(0.30-0.80)$ & . & High orange juice intake \\
\hline & & & HR $0.55(0.32-0.92)$ & . & High vegetable protein intake \\
\hline & & & HR $0.45(0.27-0.76)$ & . & High fiber intake \\
\hline & & & HR $0.54(0.32-0.90)$ & . & High bread intake \\
\hline & & & HR $0.50(0.31-0.81)$ & . & High ascorbic acid intake \\
\hline & & & Log-rank & 0.02 & $\begin{array}{l}\text { Olive oil } v s \text { butter consumption, } \\
\text { survival }\end{array}$ \\
\hline \multicolumn{6}{|l|}{$\begin{array}{l}\text { Recurrence/ Specific } \\
\text { mortality }\end{array}$} \\
\hline \multirow[t]{9}{*}{ Female breast } & Chlebowski RT et al. (2006) ${ }^{46}$ & $\mathrm{RCT} *$ & HR $0.76(0.60-0.98)$ & 0.034 & Recurrence \\
\hline & Gold EB et al. (2009) ${ }^{47}$ & $\mathrm{RCT} *$ & HR $0.69(0.51-0.93)$ & 0.02 & Recurrence \\
\hline & Shu XO et al. (2009) ${ }^{54}$ & Cohort & HR $0.68(0.54-0.87)$ & . & Recurrence \\
\hline & Guha $\mathrm{N}$ et al. (2009) ${ }^{53}$ & Cohort & HR $0.48(0.21-0.79)$ & 0.008 & Recurrence \\
\hline & McCann S et al. (2003) ${ }^{86}$ & Cohort & HR 0.29 (0.11-0.76) & trend 0.01 & Specific cancer mortality \\
\hline & Belle FN et al. (2011) ${ }^{88}$ & Cohort & $\begin{array}{ll}\text { HRR } & 0.68 \\
(0.27-1.70) & \end{array}$ & . & Specific cancer mortality \\
\hline & Borugian MJ et al. (2004) ${ }^{52}$ & Cohort & RR $4.8(1.3-18.1)$ & . & $\begin{array}{l}\text { High fat intake in postmenopausal } \\
\text { women }\end{array}$ \\
\hline & & & RR $0.4(0.2-0.8)$ & . & High protein intake \\
\hline & Kang X et al. (2010) ${ }^{55}$ & Cohort & RR 0.67 (0.54-0.85) & trend 0.02 & $\begin{array}{l}\text { Soy food intake, postmenopausal } \\
\text { women }\end{array}$ \\
\hline \multirow[t]{2}{*}{ Colorectal } & Meyerhardt JA et al. (2007) ${ }^{48}$ & Cohort & HR 3.25 (2.04-5.19) & $\begin{array}{l}\text { trend } \\
<0.001\end{array}$ & Disease free survival-western diet \\
\hline & & & HR 2.85 (1.75-4.63) & $\begin{array}{l}\text { trend } \\
<0.001\end{array}$ & Recurrence free survival \\
\hline
\end{tabular}

\footnotetext{
* Randomized clinical trial.
} 
Table 5. Studies dealing with physical activity (PA) by end-point and cancer site.

\begin{tabular}{|c|c|c|c|c|c|}
\hline $\begin{array}{l}\text { End Point } \\
\text { Cancer Site }\end{array}$ & Author (year) & Study design & Estimate $(95 \% \mathrm{CI})$ & $p$-value & Note \\
\hline $\begin{array}{l}\text { Physical Activity } \\
(P A)\end{array}$ & & & & & \\
\hline \multicolumn{6}{|l|}{ All causes mortality } \\
\hline \multirow[t]{8}{*}{ Female breast } & \multirow[t]{2}{*}{ Irwin ML et al. $(2008)^{56}$} & \multirow[t]{2}{*}{ Cohort } & HR $0.33(0.15-0.73)$ & 0.046 & $\begin{array}{l}\text { Women active } 2 \text { years after } \\
\text { diagnosis }\end{array}$ \\
\hline & & & HR $0.45(0.22-1.38)$ & . & Increased PA after diagnosis \\
\hline & \multirow{3}{*}{ Irwin ML et al. (2011) ${ }^{57}$} & \multirow{3}{*}{ Cohort } & HR 3.95 (1.45-10.5) & . & Decreased PA after diagnosis \\
\hline & & & HR $0.54(0.38-0.79)$ & $<0.01$ & High PA after diagnosis \\
\hline & & & HR $0.67(0.46-0.96)$ & . & Increased PA after diagnosis \\
\hline & Chen X et al. $(2011)^{58}$ & Cohort & HR $0.70(0.56-0.88)$ & . & $\begin{array}{l}\text { Exercise during } 36 \text { months after } \\
\text { diagnosis }\end{array}$ \\
\hline & $\begin{array}{l}\text { Ibrahim EM and } \\
\text { Al-Homaidh A (2011) }\end{array}$ & \multirow[t]{2}{*}{ Meta-analysis } & HR $0.59(0.53-0.65)$ & $\begin{array}{l}<0.0000 \\
1\end{array}$ & PA after diagnosis \\
\hline & & & HR $0.36(0.12-1.03)$ & 0.06 & PA after diagnosis, ER + tumors \\
\hline Prostate & Kenfield AS et al. $(2010)^{62}$ & Cohort & HR $0.65(0.52-0.82)$ & . & Physically active men \\
\hline \multicolumn{6}{|l|}{$\begin{array}{l}\text { Recurrence/ } \\
\text { Specific mortality }\end{array}$} \\
\hline \multirow[t]{7}{*}{ Female breast } & Holmes MD et al. $(2005)^{60}$ & Cohort & RR 0.60 (0.40-0.89) & $\begin{array}{l}\text { trend } \\
0.004\end{array}$ & Breast cancer mortality \\
\hline & Holick CN et al. $(2008)^{61}$ & Cohort & HR $0.51(0.29-0.89)$ & $\begin{array}{l}\text { trend } \\
0.05\end{array}$ & Breast cancer mortality \\
\hline & Irwin ML et al. $(2011)^{57}$ & Cohort & HR $0.61(0.35-0.99)$ & $<0.0049$ & High PA after diagnosis \\
\hline & Chen X et al. $(2011)^{58}$ & Cohort & HR $0.60(0.47-0.76)$ & . & $\begin{array}{l}\text { Exercise during } 36 \text { months after } \\
\text { diagnosis }\end{array}$ \\
\hline & $\begin{array}{l}\text { Ibrahim EM and } \\
\text { Al-Homaidh A (2011) }\end{array}$ & \multirow[t]{3}{*}{ Meta-analysis } & HR $0.66(0.57-0.77)$ & $\begin{array}{l}<0.0000 \\
1\end{array}$ & $\begin{array}{l}\text { PA after diagnosis, breast cancer } \\
\text { death }\end{array}$ \\
\hline & & & HR $0.76(0.66-0.87)$ & $\begin{array}{l}<0.0000 \\
1\end{array}$ & PA after diagnosis, recurrence \\
\hline & & & HR $0.50(0.34-0.74)$ & 0.0005 & PA after diagnosis, ER + tumors \\
\hline Colorectal & Haydon AM et al. (2006) ${ }^{62}$ & Cohort & HR $0.73(0.54-1.00)$ & . & Disease-specific survival \\
\hline Prostate & Kenfield AS et al. (2010) ${ }^{60}$ & Cohort & HR $0.88(0.52-1 \cdot 49)$ & & Physically active men \\
\hline
\end{tabular}

Several single nutritional factors have been studied (Table 4), finding lower hazard risks of overall mortality and breast cancer-specific mortality for diets with high consumption of fruits $[49,50]$ fiber $[50]$ and protein $[51,52]$. Soy food and isoflavones are protective factors for female breast cancer, and their consumption by these patients not only has a positive influence on overall mortality $[53,54]$ but can also reduce recurrence, especially in postmenopausal women [55] or those classified in higher quartiles of intake and with estrogen receptor- positive breast cancer [54]. Results were similar for overall mortality in patients with cancer of the larynx, regarding diets with high bread, orange juice, protein, fiber and ascorbic acid intake [44].

Another factor that seems to be related to better overall and cancer-specific survival in breast cancer [56-61] prostate cancer [62] and colorectal cancer [63] patients is physical activity (Table 5).

\section{Discussion}

This review found that risk and protective factors can also act as prognostic factors. The review was qualitative and only examined positive studies so the fact that it did not include negative ones could lead to some form of bias. However, two important aspects are worth stressing: 1) the search on digital databases to collect articles did not produce any completely negative studies on this topic; 2) the aim of the review was not to provide quantitative meta-analytic results.

On the other hand, some studies included in the review do not indicate the same effect in all subgroups, as in the case of soy food intake in women with estrogen-negative receptor breast cancer. In addition, the review was designed exclusively to cover qualitative outcomes on the links between risk factors and survival in cancer patients, and to 
verify the concordance between epidemiologic data and their biological explanation [4].

The epidemiological evidence indicates a consistent adverse effect of continuing smoking on lung, head and neck and bladder cancer survival. Focusing on the causal relation between cigarette smoking and several pathologies (particularly cardiovascular diseases), the effect on overall survival might simply reflect the benefits of stopping smoking in terms of a smaller reduction in mortality from cardiovascular causes. However, the expected total effect is much smaller than that observed using life table modeling, so it is likely that the longer survival is due to a reduced cancer progression [21]. In addition, the higher risk in patients who do not stop smoking at cancer diagnosis could be related to the fact that smoking increases the toxicity of the treatment, resulting in a high rate of treatment breaks and subsequent worse survival. However, this does not seem to fit with the observation that continuing smokers do not have a higher incidence of toxicity-related treatment breaks though those who did stop treatment had shorter overall survival [18].

Similar adverse effects on overall and/or disease-free survival are described for patients who continue alcohol consumption after diagnosis of head and neck cancer and those who continue to be exposed to electromagnetic fields after diagnosis of childhood leukemia. Interestingly, overall and disease-free survival was better in women consuming a high soy/isoflavone diet (especially for the estrogen receptor-positive subtype), after diagnosis of breast cancer; these habits appear to be favorable prognostic factors [49-55]. We interpret the results on recurrence or disease-free survival as a result of the slowing of cancer progression rather than as a positive effect on cardiovascular mortality due to these protective habits. Results were similar for colorectal cancer.

It is clear that caution is needed when dealing with the possible prognostic value of food habits. The evidence of their protective role in cancer patients seemed clear in this set of studies; however, in view of the very small number of studies the correlation calls for further exploration. Nonetheless, these findings suggest that a protective diet after a breast or colorectal cancer diagnosis can improve the prognosis, supporting the hypothesis that cancer progression can be affected not only by medical treatments and individual responses but also by environmental factors.

In addition, post-diagnostic physical activity seems to improve breast, prostate and colorectal survival, most of all in terms of recurrence and disease-specific mortality. This suggests that physical activity may actually affect cancer progression, in line with the results of protective dietary habits.

A further question is how to translate the epidemiological findings into a coherent discussion of their biological value. It is worth noting that the debate on cancer biology within the scientific community is still wide open [64].

The dominant theory of carcinogenesis posits that cancer is a cell based disease of the control of cell proliferation [65]. In the late 1990 s, alternative theory proposed $[4,5,66,67]$ that cancer is a tissue-based problem akin to altered histogenesis, due to changes in the interactions among different cell types, such as cells in the parenchyma and stroma. The malignant transformation would be the evolutionary cost that multicellular organisms have to pay because of their ability to develop a structured body by controlling cell differentiation and multiplication.

This perspective on carcinogenesis is usually presented under the acronym TOFT, and focuses on a different approach to cancer initiation and progression from the traditional ones. Several lines of evidence about the development of metazoans converge on the fundamental insights supported by TOFT, showing that, for example, relaxation of the negative control of cell proliferation by the tissue upon its cells is a milestone in the malignant transformation of phenotypes [68-70]. The TOFT posits that proliferation is the default state of all cells, from the ancestral ones to the ones living today. While bacteria multiply maximally in the presence of an abundant supply of nutrients, cells in metazoans are prevented from proliferating maximally by the constraints imposed on them by the tissue and organism of which they are part.

The phrase 'default state' is used to mean the ancestral ability of cells of unicellular eukaryotes to proliferate in a potentially unlimited way when nutrients are available. According to TOFT and current evolutionary biology, in metazoans this ability of proliferating spontaneously is restrained by cell-to-cell and cell-matrix signals [71]. Cells are sometimes just not inhibited enough by their neighbors, which is why normal epithelial cells can organize into a cancer when injected into a stroma that has been exposed to a carcinogen, and conversely, cells isolated from a cancer are normalized by a normal stroma [12]. The altered signaling system is a key requirement for this transition and the morpho-functional disruption of tissue organization is the most important effect.

According to the SMT, carcinogenesis is a single-cell-driven process based on mutations in growth-promoting oncogenes and in growth-suppressing genes. This theory also assumes that the default state of normal cells is quiescence [72,73]. As we can see, therefore, TOFT and SMT differ substantially in their explanations of cancer development [74], providing important arguments for epidemiological studies (Figure 1).

Another systemic perspective of carcinogenesis states that cell phenotypes are 'attractor states' within morphogenetic fields [75-77]. In other words, their phenotypic fate tends to move toward more probable states (attractors) which in turn depend on interactions within cell complexes. These cell complexes produce 'supra-cellular' structures with new collective properties, or 'emergent properties', not observed in single cells. When perturbations occur in cell complexes, they take a new course toward a new attractor state, possibly pro-cancerous. A key point of this approach to cancer biology is that it assigns a major role to the relationship between micro-environment (inter-cellular and residing within the organism) and macro-environment (outside the 
organism), and on how this effects tissue organization.

Mis-regulation of these dynamics due to toxic or other factors can disrupt the signaling and developing systems. From this viewpoint, carcinogens can promote neoplastic transition by altering the interactions between cells and their micro-environment [78]. Not surprisingly, malignant cells tend to ignore signals for regulating cell growth as they freely express the 'default state' and proliferate excessively. Currently there is debate about the role of apoptosis in cancer development. It seems that inhibition of apoptosis is not a "hallmark" of cancer [13].

According to the TOFT, tissue remodeling plays a vital role in carcinogenesis. Tissue remodeling, like in inflammation, alters the stroma-epithelium interactions and this could result in changes in tissue organization. Excess remodeling may cause a normal tissue to develop into a hyperplasia, metaplasia, dysplasia and neoplasia.

For instance, toxic exposures may trigger tissue remodeling, and this could lead to altered tissue interactions and the development of abnormal structures. In most cases, these altered states are reversible, but when remodeling lasts a long time because of persistent exposures to certain chemicals or other factors, a vicious circle can be triggered.

The long duration of an altered milieu can induce up-regulation of enzymes such as matrix metallo-proteinases by stromal fibroblasts, exacerbating inflammatory processes. If inflammation becomes chronic the extracellular matrix (ECM) tends to break up, while the shape, cytoskeleton and polarity of cells can be greatly altered by physical and chemical changes in the stromal environment. These effects are expressed basically in terms of mis-regulation and disruption at tissue level. Consequently cells tend to express their 'default states' of proliferation and motility, thus moving outside their primary site to invade the extracellular matrix, and colonize new tissues and organs by traveling across vascular structures. The organism is therefore continuously solicited to expand the primary tumor or recruit new potential 'hotspots' of carcinogenesis. This provides an important confirmation that cancer is a complex condition occurring at a higher level of biological organization than just in the cell [71,75,79-81].

This point is consistent with some of the findings of this review. For example, epidemiological observations that the use of non-steroidal anti-inflammatory drugs can reduce the risk of lung cancer, most evidently in men and for adenocarcinoma [82], point to a decisive role of chronic inflammation in cancer development. Additionally, Heymach and colleagues (2011) suggest that a low-fat diet can reduce plasma levels of specific inflammatory cytokines and angiogenic factors, slowing the progression of prostate cancer in men [83]. Other studies suggest that changes in inflammatory and/or angiogenic factors in plasma, produced by diet and physical activity, can inhibit cell proliferation in prostate tumors $[84,85]$.

We believe that the data on survival and quality of life presented in this review are in line with the TOFT approach and confirm the importance of non-genetic risk factors in producing information of prognostic value for cancer patients.

Negative effects of persistent exposure to carcinogens after a cancer diagnosis might indefinitely maintain an altered and even a pro-cancerous micro-environment within target sites. Only few studies have explored this issue in depth, revealing two points that need to be investigated. The first is the relationship between hazardous (or protective) exposure and prognosis, which could inform public policy and cancer prevention. The second is the need to explore the biological mechanisms linking cancer risk factors and prognostic factors.

\section{Conclusion}

Evidence and arguments presented in this review can be summarily considered an indication of the feasibility of environment-based cancer prevention at different stages. Cancer is a complex disease, and our understanding of its dynamics is far from complete. The basic contribution of the interaction between the micro- and macro-environment in tumor progression is only now starting to be appreciated.

The traditional paradigm of cancer looks at carcinogenesis as a process depending on a single chemical event occurring within a cell and evolving through a cascade of effects until the disease appears, except in cases whose clinical consequences never become manifest. This review's findings question that paradigm and suggest that action on environmental exposures could change the fate and the quality of life of cancer patients in particular.

Cancer dynamics occur on a higher biological scale than single cells. There is growing evidence that carcinogenesis can develop as a consequence of collective phenomena involving tissue structures. The first step is probably some change in the interactions between cells as a possible effect of detrimental ecology on the link between organism and environment. Moreover, malignant cells can reverse their malignant phenotype in response to changes in the tissue micro-environment: an important demonstration that, in cancer systems, higher structures (the tissues) can reverse their units (the cells), but not vice versa.

Strengthening these results with new evidence could be an important task for the advancement of cancer biomedicine and public health policies, and for new steps in the "war against cancer". We believe that interdisciplinary studies integrating epidemiologic, ecologic and biologic approaches are necessary to accomplish these goals.

\section{Conflict of Interest Statement}

The authors declare no conflict of interest. 


\section{REFERENCES}

[1] Baker SG, Cappuccio A, Potter JD. Research on Early-Stage Carcinogenesis: Are We Approaching Paradigm Instability? J Clin Oncol. 2010; 28(20):3215-3218.

[2] Weinberg RA. One Renegade Cell: How Cancer Begins, Basic Books, New York, 1998.

[3] Mally A, Chipman JK. Non-genotoxic carcinogens: early effects on gap junctions, cell proliferation and apoptosis in the rat. Toxicology 2002; 180:233-248.

[4] Soto AM, Sonnenschein C. The tissue organization field theory of cancer: A testable replacement for the somatic mutation theory. BioEssays, 2011; 33: 332-340.

[5] Sonnenschein C, Soto AM. The society of cells: cancer and control of cell proliferation. Springer Verlag, New York, 1999.

[6] Maffini MV, Calabro JM, Soto AM, Sonnenschein C. Stromal regulation of neoplastic development: Age-dependent normalization of neoplastic mammary cells by mammary stroma. Am J Pathol 2005; 167:1405-1410.

[7] Weaver VM, Petersen OW, Wang F, Larabell CA, Briand P, Damsky C, et al. 1997. Reversion of the malignant phenotype of human breast cells in three dimensional culture and in vivo by integrin blocking antibodies. J Cell Biol, 137, 231-245.

[8] Krause S, Maffini MV, Soto AM, Sonnenschein C, 2010. The microenvironment determines the breast cancer cells' phenotype: organization of MCF7 cells in 3D cultures. BMJ Cancer, 10, 263-270.

[9] Pierce GB, 1983. The cancer cell and its control by the embryo. Am J Pathol, 113, 116-327.

[10] Ingher DE, 2009. Can cancer be reversed by engineering the tumour microenvironment? Semin Cancer Biol, 18, 356-364.

[11] Condon MS, 2005. The role of the stromal microenvironment in prostate cancer. Seminars in Cancer Biology 15, 2, 132-137.

[12] Bussard KM, Boulanger CA, Booth BW, Bruno RD, Smith $\mathrm{GH}, 2010$. Reprogramming human cancer cells in the mouse mammary gland. Cancer Res 70:6336-6343.

[13] Enderling H, Hahnfeldt P, 2011. Cancer stem cells in solid tumors: Is 'evading apoptosis' a hallmark of cancer? Prog Biophys Mol Biol. 2011;106:391-399.

[14] Johnston-Early A, Cohen MH, Minna JD, Paxton LM, Fossieck BE Jr, Ihde DC, et al. 1980, Smoking abstinence and small cell lung cancer survival. An association. JAMA $14 ; 244(19): 2175-9$

[15] Saito-Nakaya K, Nakaya N, Fujimori M, Akizuki N, Yoshikawa E, Kobayakawa M, et al. . 2066, Marital status, social support and survival after curative resection in non-small-cell lung cancer. Cancer Sci. 97(3):206-13.

[16] Chen J, Jiang R, Garces YI, Jatoi A, Stoddard SM, Sun Z, et al. 2010. Prognostic factors for limited-stage small cell lung cancer: a study of 284 patients. Lung Cancer. 2010;67(2):221-6.

[17] Baser S, Shannon VR, Eapen GA, Jimenez CA, Onn A, Lin E, et al. 2006, Smoking cessation after diagnosis of lung cancer is associated with a beneficial effect on performance status. Chest. 130(6):1784-90.

[18] Videtic GM, Stitt LW, Dar AR, Kocha WI, Tomiak AT, Truong PT, et al. 2003, Continued cigarette smoking by patients receiving concurrent chemoradiotherapy for limited-stage small-cell lung cancer is associated with decreased survival. J Clin Oncol. 21(8):1544-9.

[19] Sardari Nia P, Weyler J, Colpaert C, Vermeulen P, Van Marck E, Van Schil P. Prognostic value of smoking status in operated non-small cell lung cancer. Lung Cancer. 2005 Mar;47(3):351-9.

[20] Videtic GM, Truong PT, Ash RB, Yu EW, Kocha WI, Vincent MD, et al. 2005, Does sex influence the impact that smoking, treatment interruption and impaired pulmonary function have on outcomes in limited stage small cell lung cancer treatment? Can Respir J. 12(5):245-50.

[21] Parsons A, Daley A, Begh R, Aveyard P. Influence of smoking cessation after diagnosis of early stage lung cancer on prognosis: systematic review of observational studies with meta-analysis. BMJ. 2010; 340:b5569.

[22] Yoshida T, Matsui K, Masuda N, Kusunoki Y, Takada M, Yana T, et al. 1996, Risk of second primary cancer in two-year survivors of small cell lung cancer. Nihon Kyobu Shikkan Gakkai Zasshi. 34(7):741-6.

[23] Tucker MA, Murray N, Shaw EG, Ettinger DS, Mabry M, Huber $\mathrm{MH}$, et al. 1997, Second primary cancers related to smoking and treatment of small-cell lung cancer. Lung Cancer Working Cadre. J Natl Cancer Inst. 3;89(23):1782-8.

[24] Kawahara M, Ushijima S, Kamimori T, Kodama N, Ogawara M, Matsui K, Masuda N, et al. 1998, Second primary tumours in more than 2-year disease-free survivors of small-cell lung cancer in Japan: the role of smoking cessation. Br J Cancer. 78(3):409-12.

[25] Kawaguchi T, Matsumura A, Iuchi K, Ishikawa S, Maeda H, Fukai S, et al. 2006, Second primary cancers in patients with stage III non-small cell lung cancer successfully treated with chemo-radiotherapy. Jpn J Clin Oncol. 36(1):7-11.

[26] Richardson GE, Tucker MA, Venzon DJ, Linnoila RI, Phelps R, Phares JC, et al. 1993, Smoking cessation after successful treatment of small-cell lung cancer is associated with fewer smoking-related second primary cancers. Ann Intern Med. 119(5):383-90.

[27] Garces YI, Yang P, Parkinson J, Zhao X, Wampfler JA, Ebbert JO, et al. 2004, The relationship between cigarette smoking and quality of life after lung cancer diagnosis. Chest. 126(6):1733-41.

[28] Chen J, Qi Y, Wampfler JA, Jatoi A, Garces YI, Busta AJ, et al. 2012, Effect of cigarette smoking on quality of life in small cell lung cancer patients. Eur J Cancer. 48(11):1593-601.

[29] Nieder C, Bremnes RM. Effects of smoking cessation on hypoxia and its potential impact on radiation treatment effects in lung cancer patients. Strahlenther Onkol. 2008 Nov;184(11):605-9.

[30] Browman GP, Wong G, Hodson I, Sathya J, Russell R, McAlpine L, et al. 1993, Influence of cigarette smoking on the efficacy of radiation therapy in head and neck cancer. $\mathrm{N}$ Engl J Med. 328(3):159-63. 
[31] Chen AM, Chen LM, Vaughan A, Sreeraman R, Farwell DG, Luu Q, et al. 2011, Tobacco smoking during radiation therapy for head-and-neck cancer is associated with unfavorable outcome. Int J Radiat Oncol Biol Phys. 79(2):414-9.

[32] Stevens MH, Gardner JW, Parkin JL, Johnson LP. Head and neck cancer survival and life-style change. Arch Otolaryngol. 1983 Nov;109(11):746-9.

[33] Do KA, Johnson MM, Doherty DA, Lee JJ, Wu XF, Dong Q, et al. 2003, Second primary tumors in patients with upper aerodigestive tract cancers: joint effects of smoking and alcohol (United States). Cancer Causes Control. 14(2):131-8.

[34] Browman GP, Mohide EA, Willan A, Hodson I, Wong G, Grimard L, et al. 2002, Association between smoking during radiotherapy and prognosis in head and neck cancer: a follow-up study. Head Neck. 24(12):1031-7

[35] Mayne ST, Cartmel B, Kirsh V, Goodwin WJ Jr. Alcohol and tobacco use prediagnosis and postdiagnosis, and survival in a cohort of patients with early stage cancers of the oral cavity, pharynx, and larynx. Cancer Epidemiol Biomarkers Prev. 2009 Dec;18(12):3368-74.

[36] Fleshner N, Garland J, Moadel A, Herr H, Ostroff J, Trambert $\mathrm{R}$, et al. 1999, Influence of smoking status on the disease-related outcomes of patients with tobacco-associated superficial transitional cell carcinoma of the bladder. Cancer. 86(11):2337-45.

[37] Chen CH, Shun CT, Huang KH, Huang CY, Tsai YC, Yu HJ, et al. 2007, Stopping smoking might reduce tumour recurrence in nonmuscle-invasive bladder cancer. BJU Int. 100(2):281-6.

[38] Kucera H, Enzelsberger H, Eppel W, Weghaupt K. The influence of nicotine abuse and diabetes mellitus on the results of primary irradiation in the treatment of carcinoma of the cervix. Cancer. 1987 Jul 1;60(1):1-4.

[39] Kwan ML, Chen WY, Flatt SW, Weltzien EK, Nechuta S, Poole EM, et al. 2012, Post-diagnosis Alcohol Consumption and Breast Cancer Prognosis in the After Breast Cancer Pooling Project. Cancer Epidemiol Biomarkers Prev. Nov 13.

[40] Greenland S, Sheppard AR, Kaune WT, Poole C, Kelsh MA. A pooled analysis of magnetic fields, wire codes, and childhood leukemia. Childhood Leukemia-EMF Study Group. Epidemiology. 2000 Nov;11(6):624-34.

[41] Foliart DE, Pollock BH, Mezei G, Iriye R, Silva JM, Ebi KL, et al. 2006, Magnetic field exposure and long-term survival among children with leukaemia. Br J Cancer. 16;94(1):161-4.

[42] Svendsen AL, Weihkopf T, Kaatsch P, Schüz J. Exposure to magnetic fields and survival after diagnosis of childhood leukemia: a German cohort study. Cancer Epidemiol Biomarkers Prev. 2007 Jun;16(6):1167-71.

[43] Miller AB, Berrino F, Hill M, Pietinen P, Riboli E, Wahrendorf J, 1994. Diet in aetiology of cancer: a review. Europ J Cancer, 30A-2,207-220.

[44] Crosignani P, Russo A, Tagliabue G, Berrino F, 1996. Tobacco and diet as determinants of survival in male laryngeal cancer patients. Int J Cancer, 65, 308-313.

[45] Kwan ML, Weltzien E, Kushi LH, Castillo A, Slattery ML, Caan BJ. Dietary patterns and breast cancer recurrence and survival among women with early-stage breast cancer. J Clin
Oncol. 2009 Feb 20;27(6):919-26.

[46] Chlebowski RT, Blackburn GL, Thomson CA, Nixon DW, Shapiro A, Hoy MK, et al. 2006, Dietary fat reduction and breast cancer outcome: interim efficacy results from the Women's Intervention Nutrition Study. J Natl Cancer Inst. 20;98(24):1767-76.

[47] Gold EB, Pierce JP, Natarajan L, Stefanick ML, Laughlin GA, Caan BJ, et al. 2009, Dietary pattern influences breast cancer prognosis in women without hot flashes: the women's healthy eating and living trial. J Clin Oncol. 20;27(3):352-9.

[48] Meyerhardt JA, Niedzwiecki D, Hollis D, Saltz LB, Hu FB, Mayer RJ, et al. 2007, Association of dietary patterns with cancer recurrence and survival in patients with stage III colon cancer. JAMA. 298(7):754-64.

[49] Ingram D. Diet and subsequent survival in women with breast cancer. Br J Cancer. 1994 Mar;69(3):592-5.

[50] McEligot AJ, Largent J, Ziogas A, Peel D, Anton-Culver H. Dietary fat, fiber, vegetable, and micronutrients are associated with overall survival in postmenopausal women diagnosed with breast cancer. Nutr Cancer. 2006;55 (2): $132-40$.

[51] Holmes MD, Stampfer MJ, Colditz GA, Rosner B, Hunter DJ, Willett WC. Dietary factors and the survival of women with breast carcinoma. Cancer. 1999 Sep 1;86(5):826-35.

[52] Borugian MJ, Sheps SB, Kim-Sing C, Van Patten C, Potter JD, Dunn B, et al. 2004 Insulin, macronutrient intake, and physical activity: are potential indicators of insulin resistance associated with mortality from breast cancer? Cancer Epidemiol Biomarkers Prev. 13(7):1163-72.

[53] Guha N, Kwan ML, Quesenberry CP Jr, Weltzien EK, Castillo AL, Caan BJ. Soy isoflavones and risk of cancer recurrence in a cohort of breast cancer survivors: the Life After Cancer Epidemiology study. Breast Cancer Res Treat. 2009 Nov;118(2):395-405.

[54] Shu XO, Zheng Y, Cai H, Gu K, Chen Z, Zheng W, Lu W. Soy food intake and breast cancer survival. JAMA. 2009 Dec 9;302(22):2437-43.

[55] Kang X, Zhang Q, Wang S, Huang X, Jin S. Effect of soy isoflavones on breast cancer recurrence and death for patients receiving adjuvant endocrine therapy. CMAJ. 2010 Nov 23;182(17):1857-62.

[56] Irwin ML, Smith AW, McTiernan A, Ballard-Barbash R, Cronin K, Gilliland FD, et al. 2008, Influence of pre- and postdiagnosis physical activity on mortality in breast cancer survivors: the health, eating, activity, and lifestyle study. J Clin Oncol. 26(24):3958-64.

[57] Irwin ML, McTiernan A, Manson JE, Thomson CA, Sternfeld B, Stefanick ML, et al. 2011, Physical activity and survival in postmenopausal women with breast cancer: results from the women's health initiative. Cancer Prev Res (Phila). 4(4):522-9.

[58] Chen X, Lu W, Zheng W, Gu K, Matthews CE, Chen Z, Zheng Y, et al. 2011, Exercise after diagnosis of breast cancer in association with survival. Cancer Prev Res (Phila). 4(9):1409-18.

[59] Ibrahim EM, Al-Homaidh A. Physical activity and survival after breast cancer diagnosis: meta-analysis of published 
studies. Med Oncol. 2011 Sep;28(3):753-65.

[60] Holmes MD, Chen WY, Feskanich D, Kroenke CH, Colditz GA. Physical activity and survival after breast cancer diagnosis. JAMA. 2005 May 25;293(20):2479-86.

[61] Holick CN, Newcomb PA, Trentham-Dietz A, Titus-Ernstoff L, Bersch AJ, Stampfer MJ, Baron JA, Egan KM, Willett WC. Physical activity and survival after diagnosis of invasive breast cancer. Cancer Epidemiol Biomarkers Prev. 2008 Feb;17(2):379-86.

[62] Kenfield SA, Stampfer MJ, Giovannucci E, Chan JM. Physical activity and survival after prostate cancer diagnosis in the health professionals follow-up study. J Clin Oncol. 2011 Feb 20;29(6):726-32.

[63] Haydon AM, Macinnis RJ, English DR, Morris H, Giles GG. Physical activity, insulin-like growth factor 1, insulin-like growth factor binding protein 3 , and survival from colorectal cancer. Gut. 2006 May;55(5):689-94.

[64] Longtin R, 2005. For Tissue Organization Theory of Cancer. A difficult road to acceptance. J Natl Cancer Inst, 97(1):11-12.

[65] Soto AM, Sonnenschein C, 2012. Is systems biology a promising approach to resolve controversies in cancer research? Cancer Cell International 2012, 12:12.

[66] Soto AM, Sonnenschein C, 2005. Emergentism as a default: Cancer as a problem of tissue organization, J Biosci, 30(1), 101-116.

[67] Sonnenschein C, Soto AM, 2011. The Death of the Cancer Cell. Cancer Res. 2011;71:4334-4337.

[68] Frisch SM, 1997. The epithelial cell default-phenotype hypothesis and its implications for cancer. BioEssays, 19:705-709.

[69] Tyler S, 2003. Epithelium-The primary building for metazoan complexity. Intergr. Comp. Biolo., 43:55-63.

[70] Johnston RN, Pai SB, Pai RB, 1992. The origin of the cancer cell: oncogeny reverses phylogeny. Biochem Cell Biol, 70(10-11):831-4.

[71] Lukashev ME, Werb Z, 1998. ECM signalling: orchestring cell behaviour and misbehavior. Trends in Cell Biology $8: 437-441$.

[72] Triolo VA, 1965. Nineteenth century foundations of cancer research advances in Enderling tumor pathology, nomenclature, and theories of oncogenesis. Cancer Res 1965, 25:76-98.

[73] Vaux DL, 2011. In defense of the somatic mutation theory of cancer. BioEssays, 33: 341-343.

[74] Parr E, 2011. The default state of the cell: quiescence or proliferation? Bioessays, 34, 36-37.

[75] Huang S, 2011. On the intrinsic inevitability of cancer: From foetal to fatal attraction. Seminars in Cancer Biology, 21, 183-199.

[76] Waddington $\mathrm{CH}$, 1957. The Strategy of the Genes: A Discussion of Some Aspects of Theoretical Biology. Allen \& Unwin, London.

[77] Huang S, Ernberg I, Kauffman S, 2009. Cancer attractors: a systems view of tumors from a gene network dynamics and developmental perspective, Semin Cell Dev Biol 20(7):869-76.

[78] Soto AM, Sonnenschein C, 2010. Environmental causes of cancer: endocrine disruptors as carcinogens. Nat Rev Endocrinol, 6(7):363-70.

[79] Bissell MJ, Radisky D, 2001. Putting tumors in context. Nature Reviews Cancer 1: 46-54.

[80] Zafira C, Tracy K, McCallister SS, 2001. The tumor macroenvironment and systemic regulation of breast cancer progression. Int. J. Dev. Biol. 55: 889-897.

[81] Bizzarri M, Giuliani A, Cucina A, D’Anselmi F, Soto AM, Sonnenschein C, 2011. Fractal analysis in a systems biology approach to cancer. Seminars in Cancer Biology 21(2011):175-82.

[82] Slatore CG, Au DH, Littman AJ, Satia JA, White E, 2009. Association of nonsteroidal anti-infiammatory drugs with lung cancer: results from a large cohort study. Cancer Epidemiol Boimarkers Prev. (4):1203-1207.

[83] Heymach JV, Shackleford TJ, Tran HT, Yoo SY, Do KA, Wergin M, et al. 2011. Effect of low-fat diets on plasma

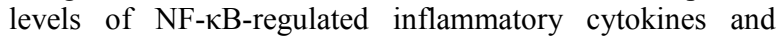
angiogenic factors in men with prostate cancer. Cancer Prev Res 4(10):1590-8.

[84] Rupnick MA, Panigrahy D, Zhang CY, Dallabrida SM, Lowell BB, Langer R, et al. Adipose tissue mass can be regulated through the vasculature. Proc Natl Acad Sci USA 2002;99:10730-5.

[85] Tymchuk CN, Barnard RJ, Heber D, Aronson WJ. Evidence of an inhibitory effect of diet and exercise on prostate cancer cell growth. J Urol 2001; 166:1185-9.

[86] McCann SE, Thompson LU, Nie J, Dorn J, Trevisan M, Shields PG, et al. 2010, Dietary lignin intakes in relation to survival among women with breast cancer: the Western New York Exposures and Breast Cancer (WEB) Study. Breast Cancer Res Treat. 122(1):229-35.

[87] Beasley JM, Newcomb PA, Trentham-Dietz A, Hampton JM, Bersch AJ, Passarelli MN, et al. 2011, Post-diagnosis dietary factors and survival after invasive breast cancer. Breast Cancer Res Treat. 128(1):229-36.

[88] Belle FN, Kampman E, McTiernan A, Bernstein L, Baumgartner K, Baumgartner R, et al. 2011, Dietary fiber, carbohydrates, glycemic index, and glycemic load in relation to breast cancer prognosis in the HEAL cohort. Cancer Epidemiol Biomarkers Prev. 20(5):890-9 